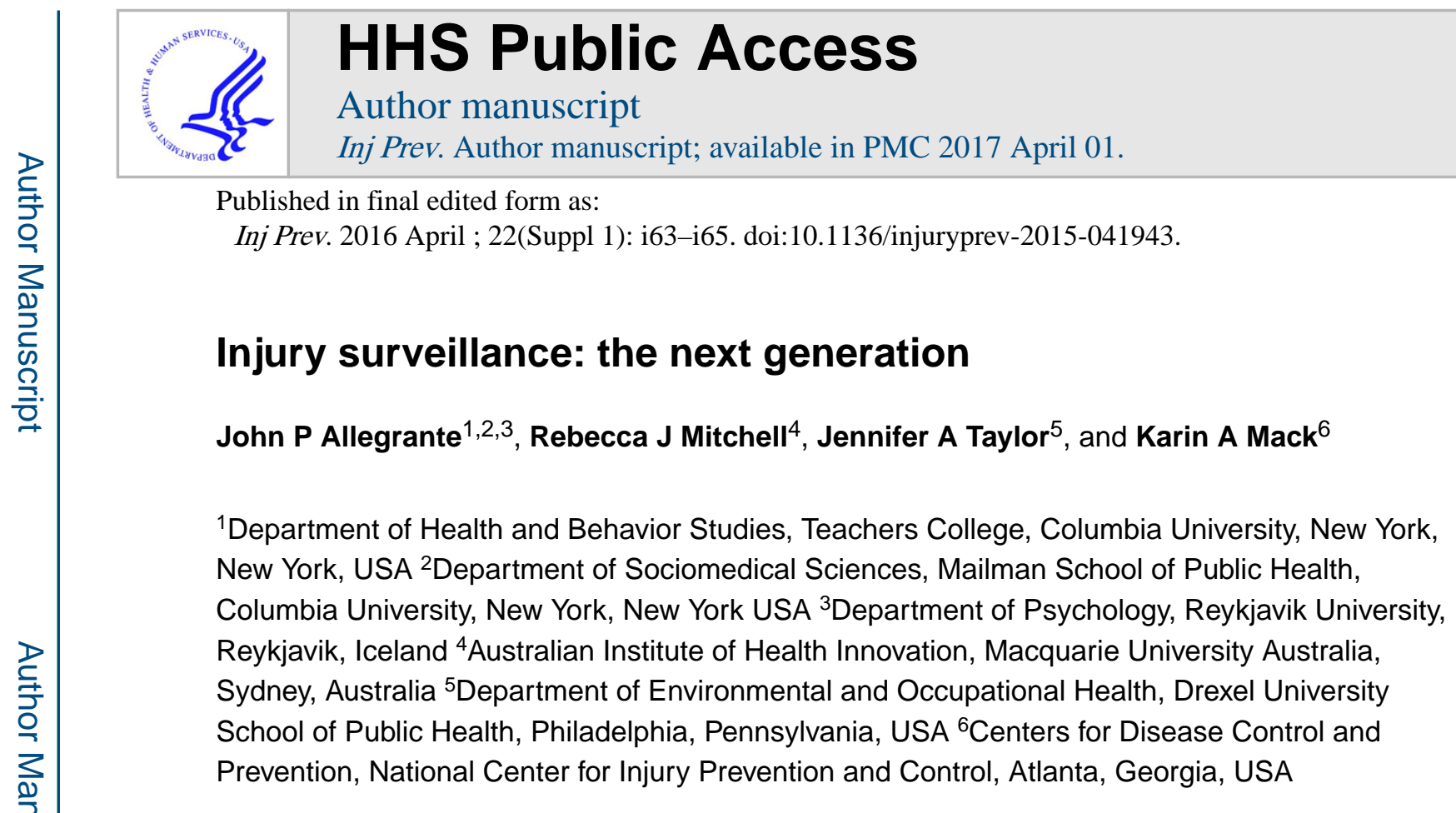

In recent years, we have seen a dramatic transformation of the knowledge economy. This transformation has been catalysed by the rapidly evolving capabilities of smart technologies and by increased recognition of the potential of using information being generated from 'big data' to empower society in a range of scientific endeavours designed to achieve the goal of improving the human condition. Former CDC Director William H Foege once wrote: "The reason for collecting, analysing, and disseminating information on a disease is to control that disease. Collection and analysis should not be allowed to consume resources if action does not follow." "The new sources of data, including big data and real-time data access, visualisation, electronic health records (eHealth), genomic risk profiling, data linkages and syndromic surveillance, have all contributed to the now-unfolding information revolution that has strengthened our public health capacity to direct and take action. Nowhere has this revolution become more apparent, nor more critical, than in the epidemiology, prevention and control of injury.

This supplement issue of Injury Prevention demonstrates that the science of surveillance has arrived as an essential element of contemporary injury prevention research and practice. The question is: how can new and ever-evolving technologies be harnessed by injury surveillance systems to achieve even better injury prevention and control benefits? The papers comprising this issue provide insights into answering this and related questions and point to the critical role new surveillance systems can play across a wide range of injury challenges.

Correspondence to John P Allegrante, Department of Health and Behavior Studies, Teachers College, Columbia University, 525 West 120th Street, Box 114, New York, NY 10027, USA; ; Email: jpa1@ columbia.edu

Contributors JPA designed and revised the paper. He is the guarantor. KAM and RJM created the initial draft and participated in revisions. JAT participated in the design and the revision of the paper.

Competing interests JPA is a member of the Board of Scientific Counselors, Centers for Disease Control and Prevention, National Center for Injury Prevention and Control. The findings and conclusions in this manuscript are those of the authors and do not necessarily represent the views of the Centers for Disease Control and Prevention.

Provenance and peer review Not commissioned; internally peer reviewed. 


\section{ACTIVE SYSTEMS}

The papers by Blair et $a l^{2}$ Chiang et $a l^{3}$ Lapidus et al and Peck et al address active systems in injury surveillance. These systems are in various stages of development, ranging from newly formed systems to well established systems whose implementation is now yielding benefits that are already being realised in understanding and reducing injury-related morbidity and mortality. They demonstrate just how far such systems have come and their remarkable adaptability across the changing nature of injuries and the changing nature of the opportunities for data collection, as well as the utility of the information that can be derived from such data. Moreover, they show how these systems can serve and be used as models for the development of surveillance systems in other areas of public health.

\section{All injuries}

For example, across all injuries, Lapidus et al guide us through the 25-year history of the Connecticut Injury Prevention Center's pioneering role in surveillance. The efforts of the Connecticut Injury Prevention Center led to important policy and legislative changes that paved the way towards a safer Connecticut. They describe the shift to new technologies that now enable injury prevention experts to analyse and disseminate information, and they demonstrate how these technologies now constitute the foundation that supports a robust state-wide fatal and non-fatal injury surveillance system that has guided research and practice, education and training, community programmes and outreach, and policy advocacy.

\section{Violence}

Changing social and cultural norms that actively or passively condone or perpetuate interpersonal violence, or both, is a long-term process, and a successful violence surveillance system must take this into consideration. The description of the National Violent Death Reporting System by Blair et a $F^{2}$ shows how critical such surveillance is for understanding the circumstances surrounding violent adult deaths. Findings from NVDRS data have been used to inform, develop, implement and evaluate violence prevention programmes that have greatly advanced injury prevention practice. The Oklahoma VDRS, for example, has revealed that $43 \%$ of homicides among women in the State of Oklahoma are related to intimate-partner violence. In Oklahoma, intimate-partner violence data from VDRS are used to inform law enforcement when managing incidents of domestic violence. Specifically, responding police officers can now conduct a brief, 11-item lethality assessment - the Lethality Assessment Protocol (LAP) — to determine if the victim is at high risk for homicide. If the results of the assessment indicate that the victim is at high risk, the officer can then facilitate immediate coordination with a local collaborating domestic violence service provider to respond. To evaluate the effectiveness of the LAP, the Oklahoma NVDRS secured a grant from the National Institute of Justice to implement and evaluate a new strategy for responding to domestic violence calls. Similarly, Chiang et al $l^{3}$ describe the Violence Against Children Surveys (VACS) as an innovative tool to collect global and national data that can provide a platform for evidence-based programming and policy reform. Although VACS in itself is not a surveillance system, the features of the surveys position VACS for use in surveillance. 


\section{Burn injuries}

In the case of burn injuries, Peck et $a I^{5}$ present a new system for gathering burns-related data. The Global Burn Registry that they describe in their article can be used in either resource-abundant or resource-limited settings. The registry was designed to reveal the aetiology of burns in these settings, provide a credible evidence base to demonstrate the public health magnitude of the issue, and serve as a tool to enable data-driven prevention programming.

\section{LINKING DATA}

The introduction and use of geographical information systems (GIS) or satellite imagery and the various GIS mapping and spatial imaging that can be generated by tools like Google Street View, together with the possibilities of linking such data to eHealth and other data sources, promise to vastly improve the capability of researchers to obtain geographical visual-display information to enhance injury surveillance. For example, the establishment of national and state-based data linkage centres in Australia has greatly advanced capacity for injury research. ${ }^{6}$ Such capacity has enabled investigators in Australia to use Google Street View together with linked police-reported crashes and hospitalisation data to compare crash patterns of novice and full-license drivers ${ }^{7}$ and to study the protective effect of roadside barriers for motorcyclists. ${ }^{8}$ Such systems have been used to provide information regarding environmental or physical conditions that may have contributed to the occurrence of an injury event, such as motor-cyclist collisions into roadside barriers, the placement of roadside infrastructure and pedestrian-vehicle collisions, and environmental circumstances that may have contributed to novice-driver crashes. Record-linkage capabilities in the era of the eHealth have, moreover, greatly enhanced the information potentially available from injury surveillance to inform injury-prevention strategies and to also guide injury-treatment practice and policies.

For example, in their report, Ising et al demonstrate real-time and cross-platform opportunities for injury surveillance in North Carolina. NC DETECT data provide a timely view into the poisoning and drug overdose burden in communities down to the Zip-code level. This capability enables communities to leverage NC DETECT data for purposes of community health assessments and subsequent intervention programme planning, including poisoning and drug overdose trends and overall rates of intentional and unintentional injuries. The range of case definitions the system permits has also allowed for the monitoring of local, emerging poisoning and drug overdose threats in near-real time.

Lyons et al ${ }^{10}$ demonstrate through the All Wales Injury Surveillance System how embedding injury surveillance within a privacy-protecting, data-linkage environment can transform the utility of a traditional, single-source surveillance system into a multisourced system. This enables a move from more passive to active surveillance, opens up innumerable opportunities to leverage resources from apparently unlinked parallel developments, and maximises the potential to engage in a wider range of research activities than the limited funds available through injury research programmes would normally allow. 


\section{NEW DATA SOURCES AND TOOLS}

Many countries have already, or are rapidly moving towards the implementation of eHealth, where individuals are able to access their own health information from a range of health agencies through one portal. Additionally, as part of the mHealth revolution, some individuals are also capturing and providing personal health and activity data through their mobile smartphones or other devices such as GPS sports watches or FitBits. These devices record the amount and type of physical activity performed and could potentially be useful in generating information on person-time risk for different types of sports activities. The use of eHealth and mHealth records to enhance injury surveillance and also to obtain injury exposure data that could be used to calculate person-time injury risk constitutes a rich source of new data that will prove invaluable to injury surveillance.

Towards that end, Bhalla and Harrison ${ }^{11}$ explain how Global Burden of Disease 2010 introduced new analytical tools, such as CODEm and DISMOD-MR, designed for constructing estimates from data aggregated from multiple sources of varying reliability. Further, they provide a simple, open-source tool for assessing the population burden of injuries, in terms of the metric disability-adjusted life years (DALYs), from estimates of the incidence of fatal and non-fatal injuries. The Burden Calculator was developed within Microsoft Excel because it is widely available and familiar to many people who are not adept at programming. This tool will allow researchers conducting descriptive epidemiological studies of injury to easily report their findings in DALYs, and thus be able to compare their results with other diseases. The tool can also be easily used to study the relative importance of various estimation parameters and thus provide guidance on how to prioritise work to improve these for better measurement.

Vallmuur et al ${ }^{12}$ show how machine learning of 'big injury narrative data' opens up possibilities for expanded sources of data that can provide more comprehensive, ongoing and timely surveillance to inform injury-prevention research, practice and policy in the future. Injury narratives provide data beyond structured coded data sets and speak of the unique nature of injuries. The authors argue for a systematic and incremental approach towards developing machine learning approaches for the specialised purpose of injury surveillance, as distinct from other applications of machine learning more broadly. Modelling techniques (and research applications) vary in terms of levels of specificity and sensitivity, simplicity and complexity, and the building and refinement of these techniques require input from content experts and technical experts. They call for the development of a big injury narrative data collaborative community to allow for the building, testing and refinement of machine-learning algorithms.

Shah and Gunn ${ }^{13}$ describe experiences of the Boston Public Health Commission in developing a syndromic surveillance system. eHealth data were used to monitor symptoms reported in chief complaints that could potentially be associated with a bioterrorism agent such as anthrax or plague. They discuss privacy, security and legal authority challenges as well as subsequent regulatory changes. 
Finally, the new world of visual analytics is the focus of the paper by Martinez et al. ${ }^{14}$ Visual analytics and data visualisation concepts and platforms could play a key role in shaping the next generation of injury surveillance. Well implemented programmes allow use of data to analyse and describe the current situation, and retrospective and predictive analysis in order to envision future injury prevention issues. As illustrated in case studies, new methods could improve data use and discovery and the analytical capacity to effectively communicate findings and key messages.

\section{WHERE ARE WE GOING AND WHAT DOES THE FUTURE HOLD?}

The potential of using real-time data collection and analysis for injury surveillance through using systems designed for syndromic surveillance-for example, BioSense 2.0 and the New South Wales Public Health Real-time Emergency Department Surveillance System ${ }^{15}$, ${ }^{16}$-is compelling. The capacity of these systems to identify different and emerging types of injuries and injury mechanisms, such as road trauma and sport-related injuries, is promising. There can also be great potential of using the range of communications and social media, including Twitter, crowd-sourcing and other tools, to contribute data in injury-surveillance efforts.

While the potential of the next generation of injury surveillance systems is great, such systems are still only in their infancy and will require significant scientific will and societal investment to further grow and mature. The challenge is in large part due to barriers to accessing big data essential for conducting injury surveillance and research that is in the public interest. ${ }^{17}$ These barriers include: accessibility of data and timely access and issues of privacy, confidentiality, and data security. It is a propitious time to examine such barriers and begin thinking about the potential strategies and partnerships to address them. While significant technological innovations have been made that can enhance injury surveillance, the above-mentioned barriers are perceived as threats to data linkage and the more granular geographical analysis that would further advance the injury research community's ability to strengthen its prevention and control efforts. Despite such challenges, the papers contained in this issue demonstrate that the global injury community is moving the needle of injury surveillance and practice to an entirely different level.

\section{References}

1. Foege WH, Hogan RC, Newton LH. Surveillance projects for selected diseases. Int J Epidemiol. 1976; 5:29-37. [PubMed: 944166]

2. Blair JM, Fowler KA, Jack SP, et al. The National Violent Death Reporting System: overview and future directions. Inj Prev. 2016; 22:i6-i11. [PubMed: 26718549]

3. Chiang L, Kress H, Sumner S, et al. Violence Against Children Surveys (VACS): an innovative, global surveillance project. Inj Prev. 2016; 22:117-i22. [PubMed: 27044493]

4. Lapidus G, Campbell B, Borrup K, et al. Practical applications of Injury Surveillance: the 25 year history of the Connecticut Injury Prevention Center. Inj Prev. 2016; 22:112-i16. [PubMed: 26728007]

5. Peck M, Meddings D, Falk H, et al. Design and implementation of a global burns repository. Inj Prev. 2016; 22:i56-i62. [PubMed: 27044496] 
6. Mitchell RJ, Cameron CM, Bambach MR. Data linkage for injury surveillance and research in Australia: perils, pitfalls and potential. Aust N Z J Pub Health. 2014; 38:275-80. [PubMed: 24890486]

7. Mitchell R, Senserrick T, Bambach M, et al. Comparison of novice and full-licenced driver common crash types in New South Wales, Australia, 2001-2011. Accid Anal Prev. 2015; 81:204-10. [PubMed: 26005055]

8. Bambach M, Mitchell R, Grzebieta R. The protective effect of roadside barriers for motorcyclists. Traffic Inj Prev. 2013; 14:756-765. [PubMed: 23944155]

9. Ising A, Proescholdbell S, Harmon K, et al. Use of syndromic surveillance data to monitor poisonings and drug overdoses in state and local public health agencies. Inj Prev. 2016; 22:i43-i49. [PubMed: 27044495]

10. Lyons RA, Turner S, Lyons J, et al. All Wales Injury Surveillance System revised: development of a population based system to evaluate single and multi-level interventions. Inj Prev. 2016; 22:i50i55. [PubMed: 26658339]

11. Bhalla K, Harrison JE. Burden calculator: a simple and open analytical tool for estimating the population burden of injuries. Inj Prev. 2016; 22:i23-i26. [PubMed: 26658340]

12. Vallmuur K, Marucci-Wellman HR, Taylor J, et al. Harnessing information from injury narratives in the 'big 'data era: understanding and applying machine learning for injury surveillance. Inj Prev. 2016; 22:i34-i42. [PubMed: 26728004]

13. Shah S, Gunn J. Big data and opportunities for injury surveillance. Inj Prev. 2016; 22:i3-i5. [PubMed: 27044494]

14. Martinez R, Ordunez P, Soliz PN, et al. Data visualization in surveillance for injury prevention and control: conceptual basis and case studies. Inj Prev. 2016; 22:i27-i33. [PubMed: 26728006]

15. Mitchell R, Bambach M. Examination of narratives from emergency department presentations to identify road trauma, crash and injury risk factors for different age groups. HIM J. 2015; 44:21-9. [PubMed: 27092466]

16. Mitchell R, Finch C, Boufous S, et al. Examination of triage nurse text narratives to identify sports injury cases in emergency department presentations. Int J Inj Contr Saf Promot. 2009; 16:153-7. [PubMed: 19941213]

17. Mitchell R, Cameron C, McClure R, et al. Data linkage capabilities in Australia: practical issues identified by a Population Health Research Network "Proof of concept project". Aust N Z J Pub Health. 2015; 39:319-25. [PubMed: 25716143] 